\title{
Train position detecting system using radio millimeter-waves
}

\author{
T. Maeda, K. Watanabe \& M. Ono \\ Central Japan Railway Company, Japan
}

\begin{abstract}
The Maglev on the Yamanashi Test Line is driven by LSM (Linear Synchronous Motor). Train control needs a precise position of the train in order to achieve stable and efficient operation of the LSM drive. Moreover, the information of the train position is important from the viewpoint of safety, because it is used to check a train speed for safety brake control that assures the last method. In the Yamanashi Maglev Test Line, a train position is detected by the inductive radio system. The inductive radio system is reliable and accurate. On the other hand, the cost of construction and maintenance of the inductive radio system is expensive, because the cross-inductive cable has to be laid along the guideway accurately and the Local Position Detectors have to be installed about every $2 \mathrm{~km}$. Thus, the train position detecting system has been developed without inductive wire in order to reduce construction and maintenance cost. It detects the train position by a combination of several methods, such as an EMF (Electromotive Force) observer, a tachometer of the wheel, a counter of ground coils for levitation and guidance, and radio millimeter-waves. In this paper, out of those detecting methods, the train position detecting system using radio millimeter-waves is presented.
\end{abstract}

Keywords: radio system, millimeter-wave, position detecting system, maglev.

\section{Introduction}

Since 1997, running tests have progressed smoothly, for over $400,000 \mathrm{~km}$ and setting a new world speed record of $581 \mathrm{~km} / \mathrm{h}$, in Yamanashi Maglev Test Line. Recently, long-term endurance test has been performed and research and development activities have been continued in order to reduce the cost of the commercial system. 
In the Yamanashi Maglev Test Line, a communication system between the Test Center and the Maglev vehicles uses an LCX (leaky coaxial cable) radio. But it is considered that the future train radio system will require a larger transmission capacity. So a radio system using millimeter-waves has been installed for experiment.

On the other hand the train position detecting system without the inductive wire has been developed in order to reduce construction and maintenance cost. It detects the train position by combination of several methods, such as an EMF (Electromotive Force) observer, a tachometer of the wheel, a counter of ground coils for levitation and guidance, and radio millimeter-waves.

One of these, the detecting system using radio millimeter-waves is difficult to achieve the same accuracy as that with the inductive wire. Nevertheless, it is able to detect the absolute position by radio propagation between the base and the mobile stations, thus it is considered that the detected position data can be utilized for block control. And it is inexpensive to introduce the position detecting system using radio millimeter-waves because an exclusive radio system is not necessary.

\section{System outline}

\subsection{Principle of detection}

The data between the mobile and the base stations is transmitted with multiflames which are sets of consecutive frames. Figure 1 shows the structure of the multiflame.

The mobile station detects a time lag between transmitter (TX) and receiver (RX) multiflame, and the information is transmitted to base stations at the particular channel time slots in the multiplex line. The base stations detect a time lag between TX and RX multiflame, and compare the time lag at the mobile station with that at the base stations, and calculate the distance between the mobile and the base stations. Then the train position is detected with the distance and the base station location. This method has been devised for being applied to independent synchronization system between the mobile and the base stations, such as the radio system using millimeter-waves in the Yamanashi Maglev test line.

Specifically, the train position is calculated by

$$
\begin{aligned}
& P_{m}=P_{b}+D \\
& D=T 3 \times c=\frac{T 2-T 1}{2} \times c \text { when } T 2>T 1 \\
& D=T 3 \times c=\frac{T 2+T m-T 1}{2} \times c \text { when } T 2 \leq T 1 \\
& (2 \times T 3<T m)
\end{aligned}
$$


where

$P_{m}$ : Position of Mobile Station

$P_{b}$ : Position of Base Station

$D$ : Distance from Base to Mobile Station

T1: Time lag between TX and RX multiframe at Mobile Station

T2: Time lag between TX and RX multiframe at Base Station

T3: Radio Propagation time

Tm: Time length of one multiframe

$c$ : Light speed

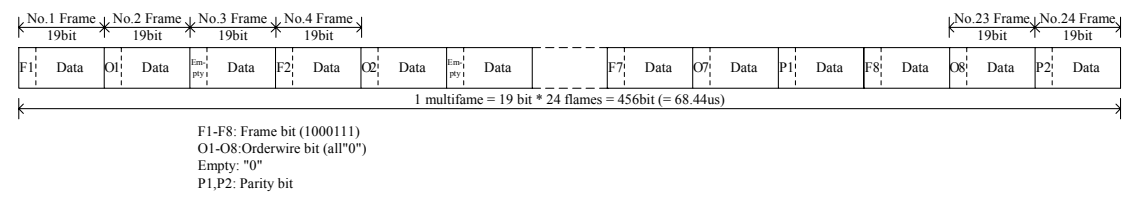

Figure 1: $\quad$ Structure of multiflame.

Figure 2 shows a chart of each multiframe. Figure 3 shows the position detecting flow.

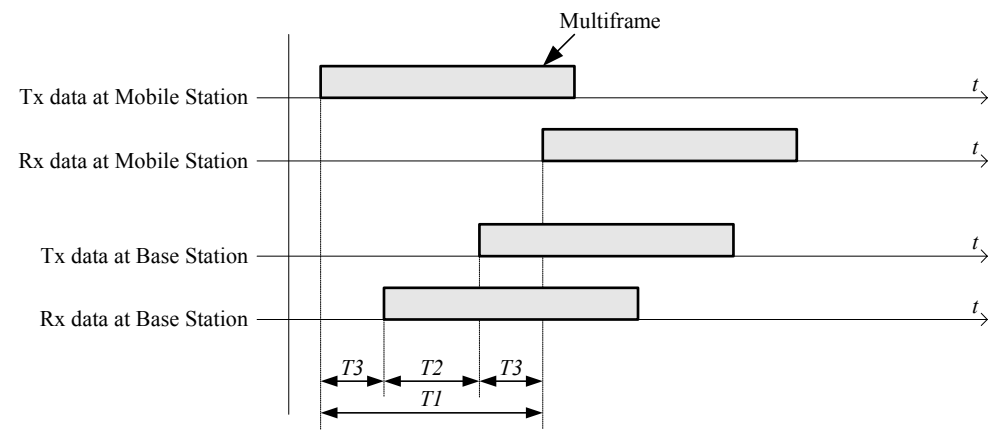

Figure 2: $\quad$ Chart of each multiframe (T2>T).

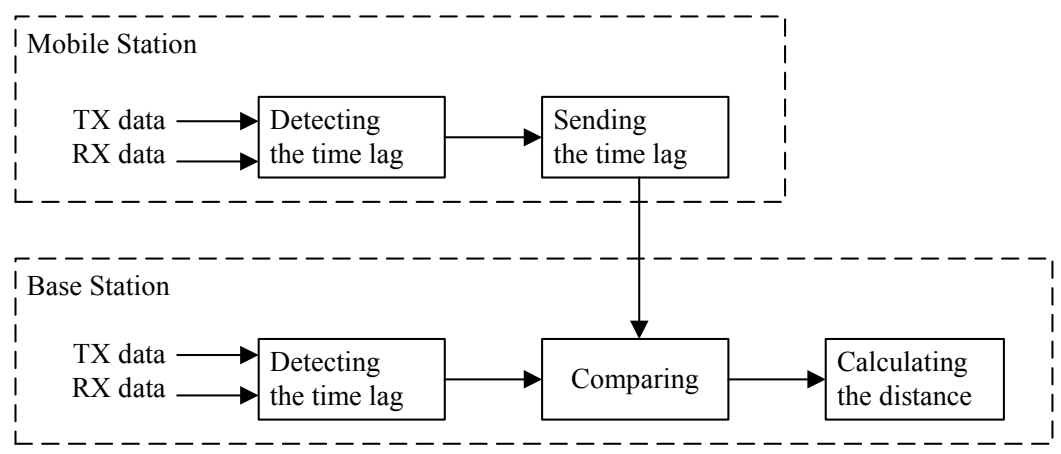

Figure 3: $\quad$ Position detecting flow. 


\subsection{Composition of position detector with radio system}

Figure 4 shows the composition of the experimental system. The position detector obtains RX multiframe from DEM (Demodulate unit) and TX multiflame from TXBB (Transmitter baseband unit) in the radio device. The position detector is connected with the radio device for the time lag data and voice transmission at the speed of $6.312 \mathrm{Mbps}$. At the base station, the position detector transmits the detected distance data to the Evaluation device. The Evaluation device obtains the standard position data from the position detector using the inductive radio system.

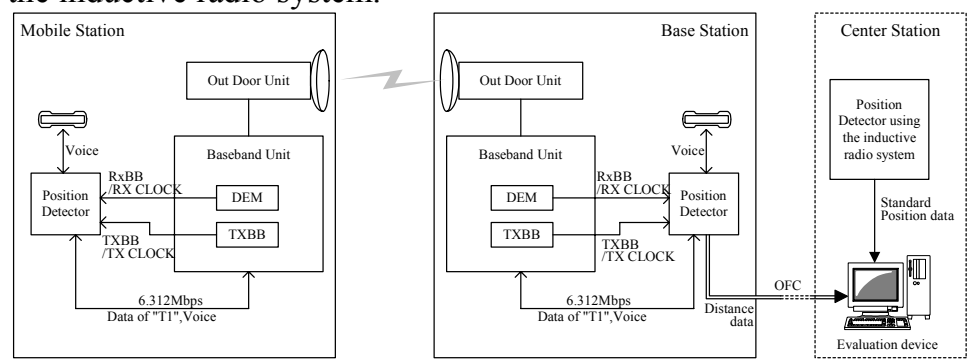

Figure 4: Composition of position detector with radio device.

\subsection{Test system configuration}

Figure 5 shows the test system configuration. In the Yamanashi Maglev Test Line, redundancy is considered in the design of the radio system using millimeter-waves. For example, any section of the test line is covered with three base stations. The base stations which communicate with the mobile station change as Maglev vehicles run. Thus, a communication between Maglev vehicles and the Test Center is kept continuously all along the test line.

In the present test line configuration, three base stations have been installed, each of which is equipped with one position detector. Thus, three kinds of position data are obtained at the same time.

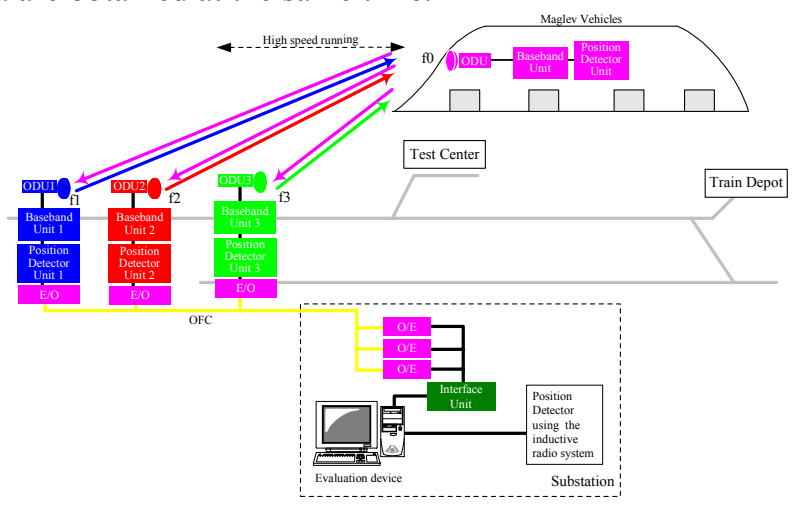

Figure 5: Test system configuration. 


\section{Test results}

\subsection{Running test results}

The position data have been acquired at high speed running tests. Figure 6 shows an example of the results. From this, it has been confirmed that it is able to detect the train position continuously from three base stations up to about $10 \mathrm{~km}$ that is the detecting limit at high speed.

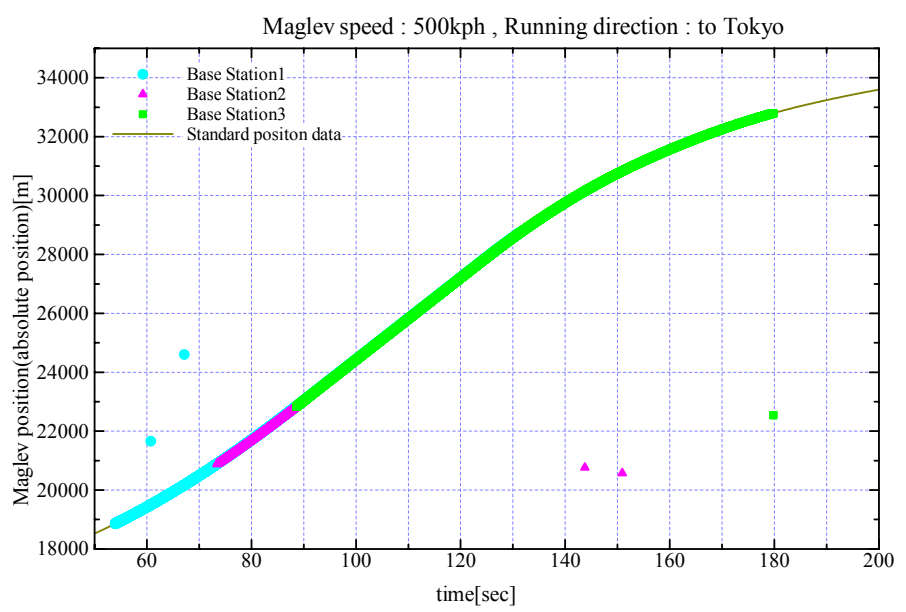

Figure 6: Example of detected position data by three base stations.

\subsection{Analyses}

\subsubsection{Rate of ineffective data}

The detected data contains extraordinary items because of transmission error or detecting fault. Here, the datum that deviates from the standard one by more than $10 \mathrm{~m}$ is defined as ineffective, and the rate of ineffective data is calculated. As a result, the rate is as low as about $2 \%$, thus it is considered that the rate level is not a problem as the position detecting tool. In addition, it has also been revealed that the rate of ineffective data does not depend on the train speed.

\subsubsection{Characteristics of position error}

It has been known that the position error increases as the mobile station goes away from the base stations. Thus, the characteristics of the position error from the standard data have been analyzed under every topography condition in the Yamanashi Maglev Test Line. The topography of the Yamanashi Maglev Test Line is described in Figure 7. Section classification in Figure 7 is explained in Table 1. The result of analysis is shown Figure 8. Consequently, a trend of the increase in position error is characterized for every topography condition. It is considered that the position data can be corrected based on the trend. 


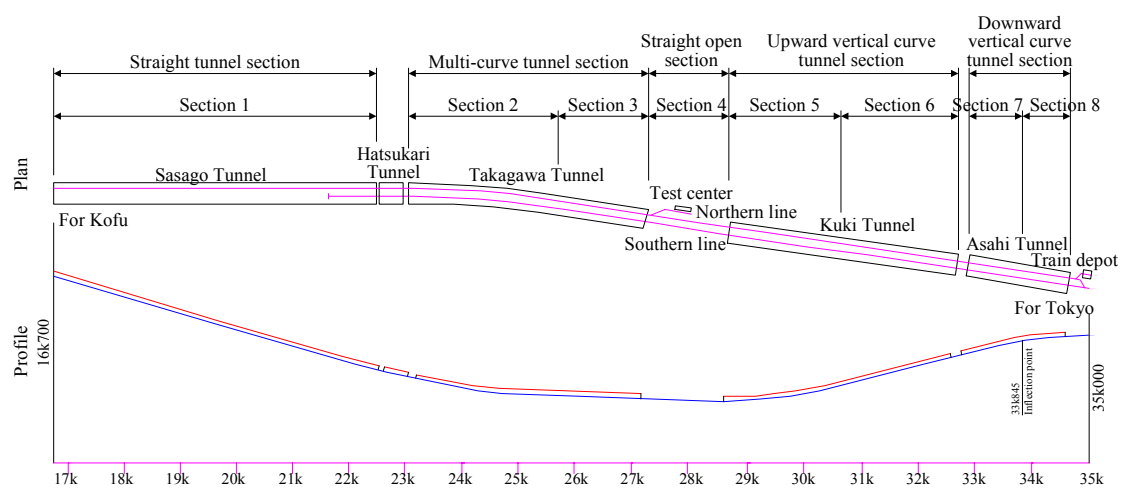

Figure 7: $\quad$ Topography of Yamanashi Maglev test line.

Table 1: $\quad$ Section classification.

\begin{tabular}{|c|l|}
\hline Section No. & Classification \\
\hline 1 & Straight tunnel section \\
\hline 2 & Multi-curve tunnel section \\
\hline 3 & Straight tunnel section \\
\hline 4 & Straight open section \\
\hline 5 & Upward vertical curve tunnel section \\
\hline 6 & Straight tunnel section \\
\hline 7 & Downward vertical curve tunnel section to inflection point \\
\hline 8 & Downward vertical curve tunnel section from inflection point \\
\hline
\end{tabular}

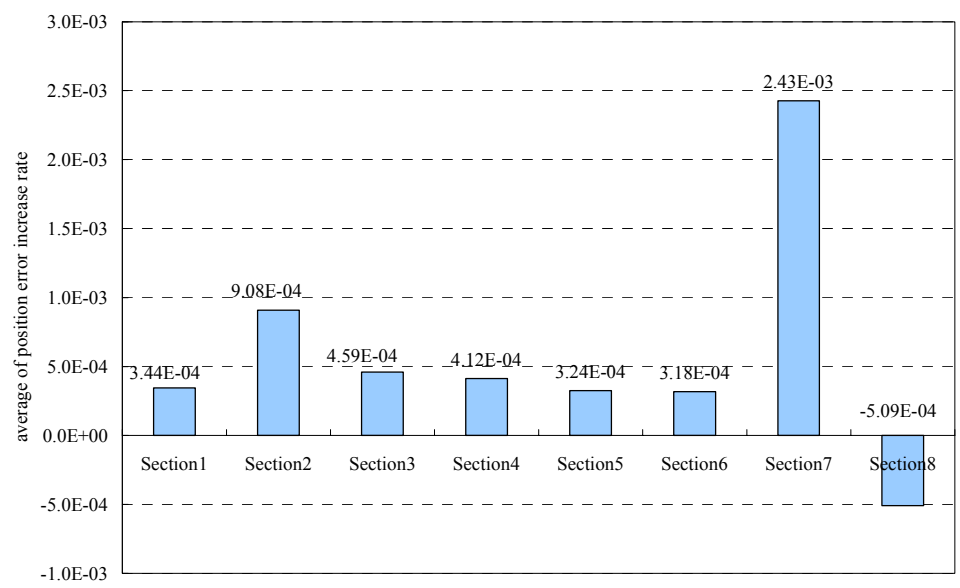

Figure 8: Characteristics of position error. 


\subsubsection{Results of processing data}

The position data have been processed by the following procedures. The example of position error data before processing is shown Figure 9, and the example of position data after processing is shown Figure 10. The extraordinary data are interpolated, and the scatter of the position error data became considerably small.

(1)Position data smoothing

The position data are smoothed by the method of least squares.

$$
\begin{aligned}
& y=a+b x \\
& a=\frac{\left(\sum y_{i}\right)\left(\sum\left(x_{i}^{2}\right)\right)-\left(\sum x_{i}\right)\left(\sum x_{i} y_{i}\right)}{k\left(\sum\left(x_{i}^{2}\right)\right)-\left(\sum x_{i}\right)^{2}} \\
& b=\frac{k\left(\sum x_{i} y_{i}\right)-\left(\sum x_{i}\right)\left(\sum y_{i}\right)}{k\left(\sum\left(x_{i}^{2}\right)\right)-\left(\sum x_{i}\right)^{2}}
\end{aligned}
$$

$k$ : population parameter $=30$

(2)Delay correcting

The position data are corrected using the delay time from the standard data.

(3)Position error correcting

The position data are corrected, using correction the data shown in Figure 8.

(4)Position data selection

The most suitable position datum is selected from three position data.

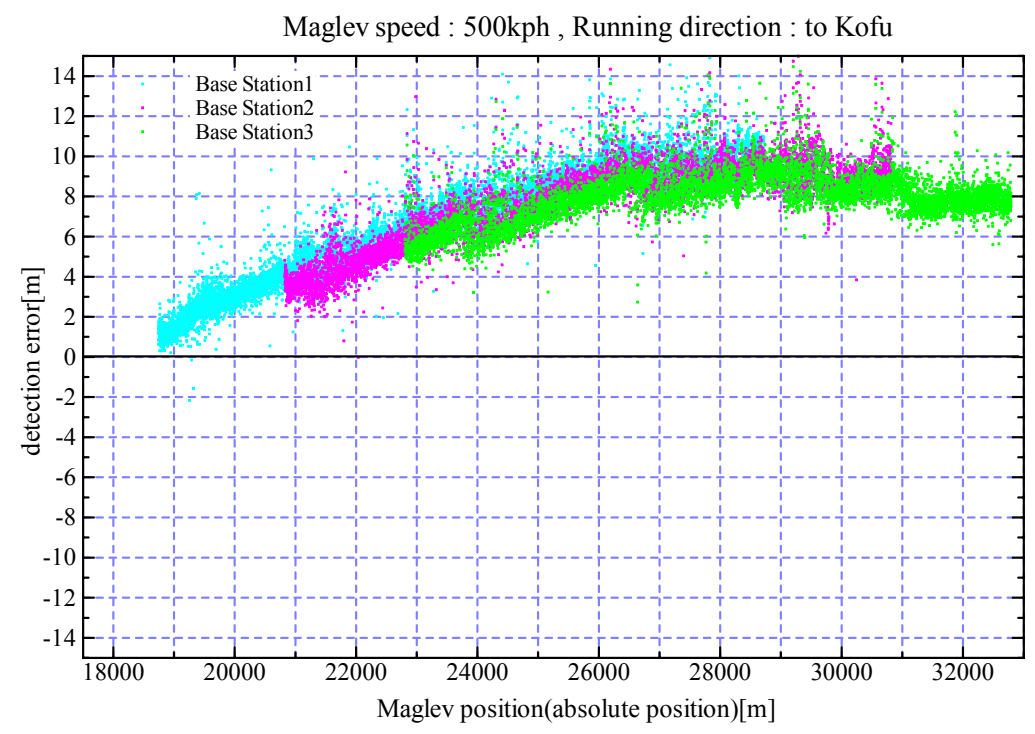

Figure 9: Example of position error before processing. 


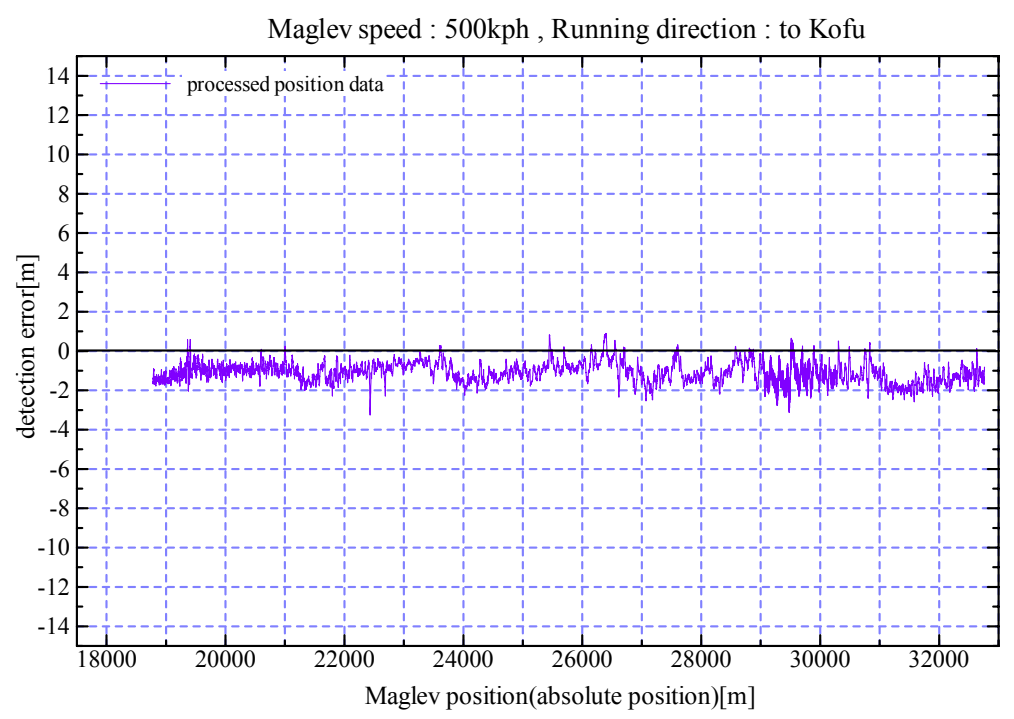

Figure 10: Example of position error after processing.

\section{Conclusion}

In the development, the knowledge of the characteristics of the position data has been obtained, and the method of processing of the position data has been established. It is considered that the system can be utilized as one of the position detecting tools.

The position detecting system will be installed along the whole Yamanashi Maglev Test Line, and will be developed aiming to realize practical use of a new position detecting system without the inductive wire.

\section{References}

[1] H. Yamamura \& S. Sasaki, Millimeter Wave Propagation Model and Delay Spread along the Maglev Guideway, IEICE TRANS COMMUN,vol.E78B,No.8,1995

[2] H. Niikura, H. Yamamura, K. Yamakawa, H. Uenae \& K. Honma, Train Radio System Using Millimeter-Waves for the Superconducting Maglev System, Maglev'95, PP405-409,1995

[3] T. Nakashima, S. Kaga \& H. Ohtsuki, Results of electrical experiments on the Yamanashi Maglev Test Line, Maglev'2000, PP89-94,2000

[4] S. Koga, J. Enomoto \& S. Kaga, Sensorless Control System of Superconducting Maglev,Maglev'2002, 2002

[5] S. Miyamoto, Y. Osada, K.Yamazumi \& T. Furuki, The Status of the running tests of JR-Maglev, Maglev'2004,PP60-64,2004 